\title{
RNB production with thermal neutrons
}

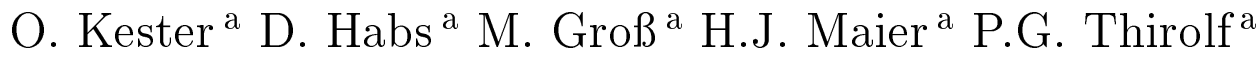 \\ T. Sieber ${ }^{a}$ T. Faestermann ${ }^{b}$ T. von Egidy ${ }^{b}$ U. Köster ${ }^{c}$ \\ ${ }^{a}$ LMU München, Sektion Physik, Am Coulombwall 1, D-85748 Garching \\ ${ }^{\mathrm{b}}$ TU München, Physik Department, D-85748 Garching \\ ${ }^{\mathrm{c}}$ ISOLDE, CERN, CH-1211 Geneva 23
}

\begin{abstract}
Thermal neutron induced nuclear fission is the most suitable method to produce neutron-rich isotopes $((70 \leq \mathrm{A} \leq 160)$ due to the large fission cross section and the high thermal neutron fluxes in modern reactors. Intensities of mass separated neutron rich nuclei of some $10^{11}$ ions/s are expected e.g. for ${ }^{91} \mathrm{Kr},{ }^{132} \mathrm{Sn}$ or ${ }^{144} \mathrm{Cs}$ from

${ }^{235} \mathrm{U}$ diluted in a porous graphite target. Several front runners with low energy fission fragment beams exist like OSIRIS in Studsvik. In order to get beams of neutron rich nuclei at the Coulomb barrier the PIAFE project worked out a first concept of production and mass separation of high intensity beams of fission fragments. At the new Munich high flux reactor FRMII the Munich Accelerator for Fission Fragments (MAFF) is under development to make use of post accelerated beams of neutron rich isotopes for experiments in many different fields of nuclear physics, solid state physics and medicine. One key experiment will be the production and the study of very heavy elements. An overview of the production method of neutron rich isotopes by thermal neutron induced fission, and of the expected yields will be given and the development of target ion sources and of the fission targets for MAFF will be characterised.
\end{abstract}

\section{Introduction}

For about fifty years thermal neutron induced fission of ${ }^{235} \mathrm{U}$ has been used for the production of radioactive nuclear beams (RNB). After first experiments at the on-line isotope separator at the Copenhagen cyclotron more than a dozen of reactor based ISOL facilities have been in operation [1]. Among reactor based facilities the highest yields and the most variety of elements are obtained by target-ion source assemblies in an in-pile arrangement as it is used at OSIRIS in Studsvik [2]. The target and ion source system is located $15 \mathrm{~cm}$ apart from the wall of the $1 \mathrm{MW}$ reactor $\mathrm{R} 2-0$. The maximum flux of

Preprint submitted to Elsevier Preprint $\quad 4$ May 2001 
$3^{*} 10^{11} \mathrm{n} / \mathrm{cm}^{2} \mathrm{~s}$ can be reduced by a factor of 100 by moving the reactor $25 \mathrm{~cm}$ further away. The idea to use neutron rich isotopes from an in-pile targetion source for experiments is an alternative to the traditional concept that radioactive ion beams are produced by energetic primary beams of charged particles via spallation, fragmentation or fission reactions. In both cases the produced radioactive nuclei are extracted as singly charged ions from an ion source, mass separated, ionised to higher charge states, and finally accelerated to energies of a few $\mathrm{A} \cdot \mathrm{MeV}$. The production via neutron induced fission has the advantage of the high fission cross section of ${ }^{235} \mathrm{U}$ for thermal neutrons of about $586 \mathrm{~b}$. The high neutron flux allows to increase the fission rate by several orders of magnitude with respect to existing accelerators. A variety of experiments benefit from the high number of neutrons of the fragments [3] and of the high expected high intensities. Two flagship experiments shall be mentioned: The production of heavy elements $(Z>100)$ via fusion reactions [4] and the high resolution nuclear spectroscopy in the vicinity of the doubly magic nuclei ${ }^{78} \mathrm{Ni}$ and ${ }^{132} \mathrm{Sn}$. These regions are close to the path of the astrophysical r-process. The fission process can approach the neutron drip-line more than those achieved by other reactions.

\section{2 cross sections and yields}

In comparison to the production cross section for neutron rich isotopes by charged particle beams the production cross section by neutron induced fission is about three orders of magnitude larger [5]. A typical neutron flux in a high flux reactor like the ILL reactor of the new FRM-II at Munich exceeds $10^{14} \mathrm{n} / \mathrm{cm}^{2} \mathrm{~s}$. Concerning an Uranium load of $1 \mathrm{~g}$ it will lead to $10^{14}$ fission $/ \mathrm{s}$ and to a heating power of $3 \mathrm{~kW}$. The power produced in the target is due to the energy release of the fission process itself, whereas with charged primary beams most of the energy is due to the energy loss of the beam. Due to the high fission cross section, a $1 \mathrm{GeV}$ proton beam for the production of a comparable amount of fission fragments from ${ }^{238} \mathrm{U}$ must exceed $50 \mathrm{~mA}$. Such intensity can not be handled by any ISOL target technology. Today's practical developments for highest power targets are in the range of intensities up to 100 $\mu \mathrm{A}$ primary proton beam and they aim mainly for the more robust tantalum foil targets [6]. It is not clear that compact actinide targets can stand these beam intensities. The production of fission fragments by fast neutrons with energies of several $\mathrm{MeV}$, suffers from the rather low fission cross section of about $3 \mathrm{mb}$. In order to get competitive numbers of radioactive nuclei about $500 \mathrm{~g}$ of ${ }^{235} \mathrm{U}$ are required. Thus a thick target is required, which leads to longer release times and to a lower release efficiency.

The production of suitable intensities of neutron rich isotopes in case of 
thermal neutron induced fission is restricted to masses between $A=70$ and $\mathrm{A}=160$, which corresponds to elements from $\mathrm{Ni}$ to $\mathrm{Eu}(\mathrm{Z}=28-63)$. Isotopes with $Z=40-46$ are refractory and can not be extracted. Furthermore they are located in the minimum of the fission yield distribution [7]. Table 1 shows the fission yields and the expected beam intensities of some elements of the light mass peak which play a major role in the key experiments mentioned above. For the production of superheavy elements via cold fusion, reaction like ${ }^{208} \mathrm{~Pb}\left({ }^{82} \mathrm{Ge}, 1 \mathrm{n}\right){ }^{290} 114,{ }^{208} \mathrm{~Pb}\left({ }^{94} \mathrm{Kr}, 1 \mathrm{n}\right){ }^{302} 118$ and ${ }^{208} \mathrm{~Pb}\left({ }^{94} \mathrm{Sr}, 1 \mathrm{n}\right){ }^{302} 120$ for instance are of interest $[4,8]$. With radioactive targets [9] even more neutron rich heavy elements could be produced with lifetimes up to years. For elements of the heavy mass peak inverse fusion reactions with double magic nuclei like ${ }^{132} \mathrm{Sn}\left({ }^{132} \mathrm{Sn}, 2 \mathrm{n}\right){ }^{260} \mathrm{Fm}$ may give the opportunity of unifying the understanding of fusion and fission. For MAFF projectile intensities of about $10^{11} / \mathrm{s}$ are expected, which are only one order of magnitude lower than intensities of stable beams presently used at GSI for the production of heavy elements [10]. Due to the high intensities the production of radioactive targets will become possible by implantation of some longer living isotopes. For the study of the nuclear r-process the intensities of waiting point nuclei in the neighbourhood of ${ }^{132} \mathrm{Sn}$ and ${ }^{78} \mathrm{Ni}$ are of main interest. Information of the shell structure of these nuclei can be obtain by Coulex, transfer reactions and decay spectroscopy. One big advantage of using fission fragments is the peaked isobaric elements distribution, which simplifies the isobaric mass separation for experiments with fission fragments near the dripline.

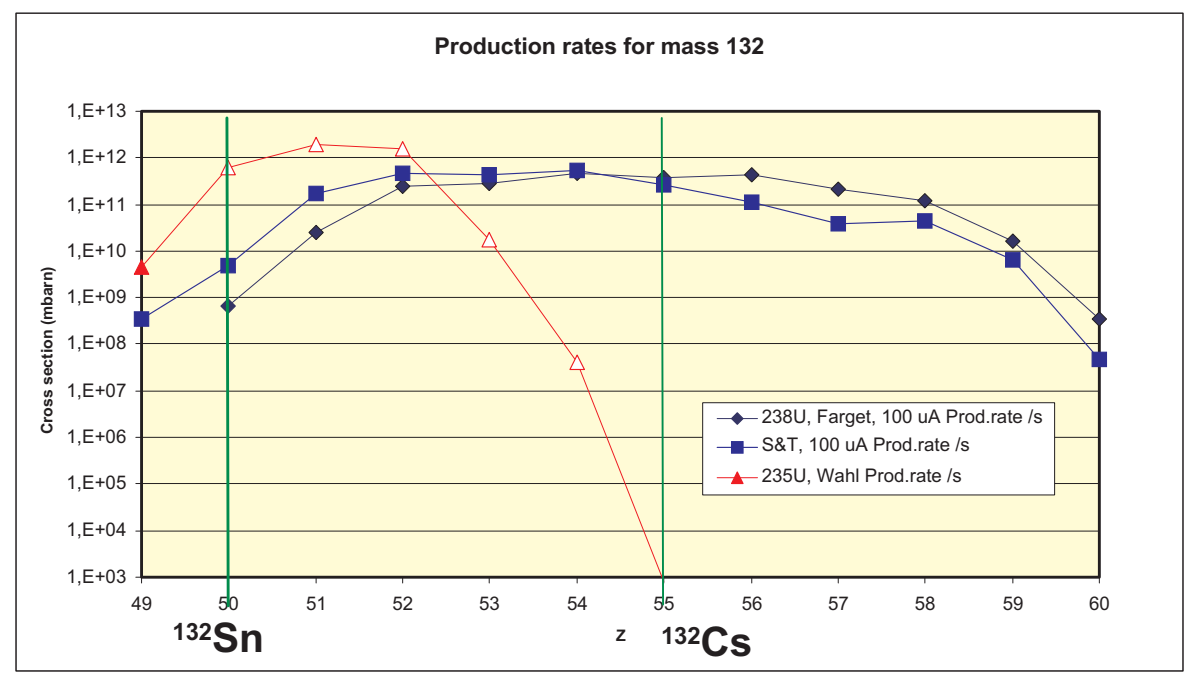

Fig. 1. Comparison of the production rates of mass $A=132$ by thermal neutron induced fission and a $100 \mu \mathrm{A} 1 \mathrm{GeV}$ p-beam derived from [7,11,12].

Fig. 1 shows a comparison of the isobaric production cross section of mass $\mathrm{A}=132$ for thermal neutron induced fission and for production with a $1 \mathrm{GeV}$ proton beam $(100 \mu \mathrm{A})$. In the case of high energy proton induced fission the abundance of the easily surface ionised isobaric contaminant ${ }^{132} \mathrm{Cs}$ is several orders of magnitude higher than the abundance of ${ }^{132} \mathrm{Sn}$. While for thermal neu- 
tron induced fission, the abundance of Cs is seven orders of magnitude lower. The same isobaric contamination problem occurs in the case of p-induced fission in the vicinity of ${ }^{78} \mathrm{Ni}$, due to the very small production yields.

Table 1

Expected yields of prominent isotopes at MAFF compared with ISOLDE experimental yields. The primary beam of ISOLDE are $2.5 \mu \mathrm{A}$ of $1 \mathrm{GeV}$ protons (or $600 \mathrm{MeV}$ protons for the SC).

\begin{tabular}{|c|c|c|c|c|c|c|}
\hline Isotope & $\begin{array}{l}\text { Prod. rate } \\
\qquad\left(\mathrm{s}^{-1}\right)\end{array}$ & $\begin{array}{c}\text { Half-life } \\
\qquad(\mathrm{s})\end{array}$ & $\begin{array}{c}\text { Release } \\
(\%)\end{array}$ & $\begin{array}{l}\text { Ioniz. } \\
(\%)\end{array}$ & $\begin{array}{l}\text { MAFF } \\
\left(\mathrm{s}^{-1}\right)\end{array}$ & $\begin{array}{l}\text { ISOLDE } \\
\left(\mathrm{s}^{-1}\right)\end{array}$ \\
\hline${ }^{78} \mathrm{Zn}$ & $2.3 \cdot 10^{9}$ & 1.47 & 36 & 10 & $8 \cdot 10^{7}$ & $1 \cdot 10^{6}(\mathrm{SC})$ \\
\hline${ }^{82} \mathrm{Ge}$ & $1.2 \cdot 10^{11}$ & 4.6 & $<48$ & 10 & $<6 \cdot 10^{9}$ & $2 \cdot 10^{5}(\mathrm{SC})$ \\
\hline${ }^{91} \mathrm{Kr}$ & $3.3 \cdot 10^{12}$ & 8.6 & 89 & 15 & $4 \cdot 10^{11}$ & $4 \cdot 10^{8}$ \\
\hline${ }^{94} \mathrm{Kr}$ & $1.1 \cdot 10^{11}$ & 0.2 & 16 & 15 & $3 \cdot 10^{9}$ & $6 \cdot 10^{6}$ \\
\hline${ }^{97} \mathrm{Rb}$ & $3 \cdot 10^{10}$ & 0.17 & 10 & 80 & $2 \cdot 10^{9}$ & $1.5 \cdot 10^{7}$ \\
\hline${ }^{94} \mathrm{Sr}$ & $4.6 \cdot 10^{12}$ & 74 & 90 & 10 & $5 \cdot 10^{11}$ & $6 \cdot 10^{8}(\mathrm{SC})$ \\
\hline${ }^{132} \mathrm{Sn}$ & $7 \cdot 10^{11}$ & 39.7 & 89 & 10 & $6 \cdot 10^{10}$ & $7.5 \cdot 10^{8}$ \\
\hline${ }^{142} \mathrm{Xe}$ & $5.2 \cdot 10^{11}$ & 1.24 & 7 & 25 & $1 \cdot 10^{10}$ & $1 \cdot 10^{7}(\mathrm{SC})$ \\
\hline${ }^{144} \mathrm{Cs}$ & $4.3 \cdot 10^{11}$ & 1.0 & 47 & 80 & $2 \cdot 10^{11}$ & $3 \cdot 10^{8}(\mathrm{SC})$ \\
\hline
\end{tabular}

\section{Target ion sources for high flux reactors}

As the ion beam intensity mainly depends on the speed and efficiency of the diffusion, effusion and extraction of the fission products, the target ion source in a high flux reactor environment is a crucial issue. In order to increase the release efficiency of the radioactive nuclei from the target, the temperature of the target ion source combination needs to be raised to high temperatures. On the other hand a fast sublimation of the target material has to be avoided. To optimize the yields the temperature has to be set for each specific element. Nevertheless a long lifetime (one reactor cycle, $52 \mathrm{~d}$ ) at high operation temperatures (up to $2400^{\circ} \mathrm{C}$ ) is required. For instance the ANUBIS source at OSIRIS in Studsvik has lifetimes of $1200 \mathrm{~h}$ at $2400{ }^{\circ} \mathrm{C}$ operation temperature [13]. In order to increase the ionisation efficiency as much as possible, dedicated sources for different elements with different ionisation potentials are required [14]. The surface ionisation source provides ionisation efficiencies of about $100 \%$ for alkaline metals. The resonant ionisation laser ion source (RILIS) has an efficiency of about $10-20 \%$ for metals with an ionisation potential of $6-9$ $\mathrm{eV}$. Due to the multistep ionisation process the RILIS has a very high element selectivity [15]. Bunching of the ions and gating of the separator can further 
reduce the background of surface ionised elements. For non-metallic elements As to $\mathrm{Kr}$ and $\mathrm{Sb}$ to Xe with very high ionisation potentials, a plasma ion source like the FEBIAD source is required [16]. Efficiencies up to $50 \%$ can be reached at very low support gas pressures of $10^{-6}$ mbar which can be delivered by radiogenic $\mathrm{Kr}$ and $\mathrm{Xe}$.

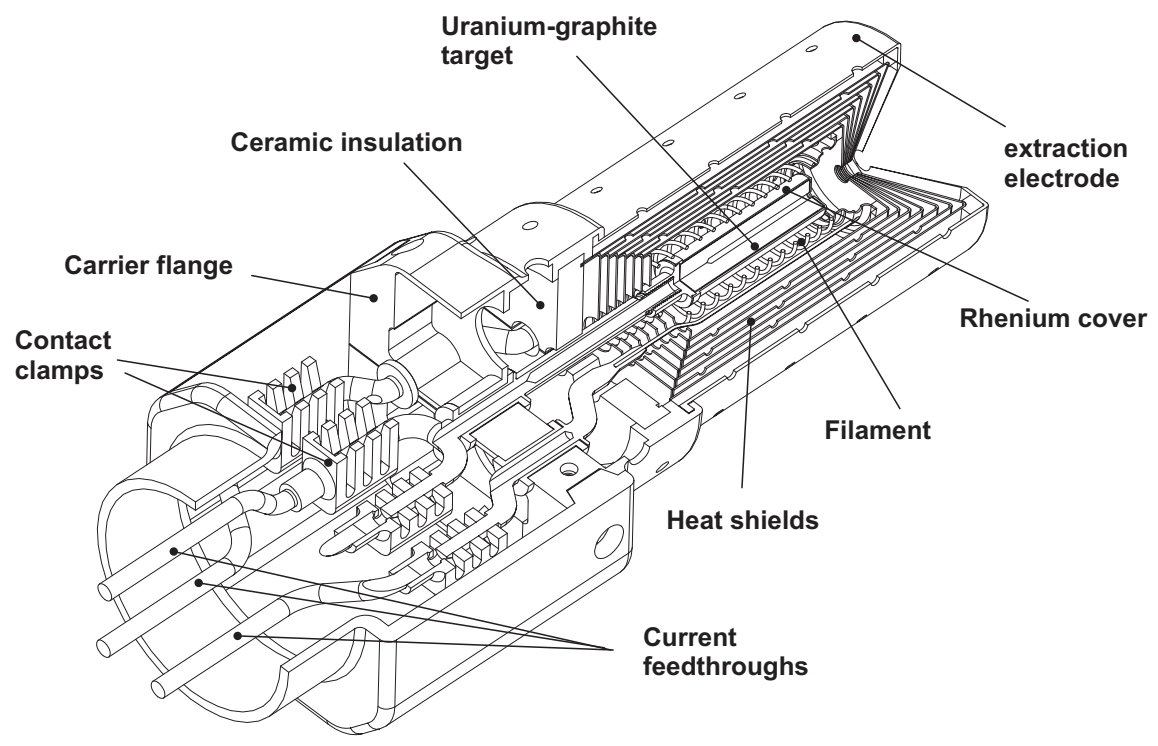

Fig. 2. Lay-out of a surface ionisation source for MAFF.

\subsection{The MAFF target ion source}

The source construction should be as simple as possible to improve the reliability in the hostile environment and to facilitate the removal and disposal of the source $(10 \mathrm{kCi}$ activity after one reactor cycle of operation and $1 \mathrm{~g} \mathrm{U}$ content). The lay-out of the surface ionisation source for MAFF is shown in Fig.2. There the front end of the supporting rod and the exchangeable target ion source are sketched. The target is surrounded from a tungsten filament, which will be used for temperature stabilisation of the target due to electron bombardment. Thus the power reduction from the U burn-up within the reactor cycle can be compensated. The heat shields made from Rhenium are only required in case of lower $U$ content in first test runs. Running with the full $\mathrm{U}$ content, only the extraction electrode and the heat shields towards the ceramic insulator will be present.

\subsection{The target design for the MAFF in-pile source}

For thermal neutrons the level of technical development in Studsvik allows the use of $1 \mathrm{~g}^{235} \mathrm{U}$ in a flux density of $3^{*} 10^{11} \mathrm{n} / \mathrm{cm}^{2} \mathrm{~s}$. Concerning the neutron 
flux in a reactor like the FRM-II, some target development is required. The rather compact target for the MAFF fission sources consists of $99 \%$ enriched ${ }^{235} \mathrm{UC}_{2}$ distributed homogeneously in a porous graphite matrix which has the shape of a cylinder of $15 \mathrm{~mm}$ diam. x $60 \mathrm{~mm}$ length with a narrow, coaxial, single-ended channel [17]. The graphite cylinder is mounted in a protecting Re container having a $2 \mathrm{~mm}$ diam. aperture in front of the channel. Re is a refractory metal with a melting point of $3180{ }^{\circ} \mathrm{C}$. It has been chosen as a construction material for the target container, because it is known to be most inert against $\mathrm{C}$ and $\mathrm{U}$ at high temperatures [18] and to withstand the high neutron flux at the target position [19]. The Re cover is an important part of the target assembly. Besides providing mechanical stability, it prevents the fissile ${ }^{235} \mathrm{U}$ and the fission fragments from escaping through the periphery of the graphite matrix, as well as excessive sublimation of graphite.

\section{Testing of MAFF $\mathrm{UC}_{2}$ targets}

As the target tests are a crucial issue for the development of a target ion source in an high flux reactor several tests have been foreseen in Munich to study different target preparation methods and to examine the chemical properties of the targets. In order to test the ion source off-line, a mass separator beam line will be built up to study the ion production and extraction capabilities. The two target preparation methods which are under examination in our technical laboratory are:

- A concentrated aqueous solution of $\mathrm{UO}_{2}\left(\mathrm{NO}_{3}\right)_{2}$ will be added to a graphite sample. Then the sample is removed from the liquid and dried in ambient atmosphere by an IR radiator, resulting in solid $\mathrm{UO}_{2}\left(\mathrm{NO}_{3}\right)_{2}$ crystals dispersed in the graphite matrix. Conversion into $\mathrm{UC}_{2}$ requires heating under vacuum at $1500-2000{ }^{\circ} \mathrm{C}$. Several graphite types of different porosity will be investigated to achieve the most suitable concentration and distribution of $\mathrm{U}$ in the graphite matrix.

- An alternate procedure is pressing a mixture of $\mathrm{UO}_{2}$ and excess graphite powders into the desired cylindrical shape and subsequent heat treating of the sample under vacuum at a temperature above $1500{ }^{\circ} \mathrm{C}$ [20].

The target assembly has to stand at least one reactor cycle of 52 days or approximately $1250 \mathrm{~h}$. Its long term behaviour has to be studied in preliminary tests to guarantee a reliable operation of the ion source during that period. Several detrimental phenomena may arise and require remedial measures or conceptional changes prior to operation in the reactor:

- Diffusion and evaporation of ${ }^{235} \mathrm{U}$, resulting in possible reduction of the yield and embrittlement of the container 
- Diffusion and sublimation of graphite, resulting in possible short circuits across insulators outside the target assembly

- Fatigue of the Re container material, including recrystallisation, formation of pores, and thermochemical reactions with $\mathrm{C}$ and $\mathrm{U}$

- Change of the emissivity of the Re container material, leading to a destabilisation of the temperature equilibrium

For simulation of the operational conditions, a vacuum furnace with a useful volume of $80 \mathrm{~mm}$ diam x $160 \mathrm{~mm}$ height has been set up (fig.3)s, which is capable to produce a temperature of $2500{ }^{\circ} \mathrm{C}$ for the required period. The power input is $40 \mathrm{~kW}$. The hot zone consists of a W mesh heater surrounded by a set of heat shields made of $\mathrm{W}$ and Mo. It is installed in a jacketed stainless steel vacuum chamber of $600 \mathrm{~mm}$ diam x $600 \mathrm{~mm}$ height, which is water cooled during operation. The temperature control is performed either by a thermocouple or, at temperatures above $2000{ }^{\circ} \mathrm{C}$, by an IR ratio pyrometer. A computer controlled power supply allows to run temperature-time programs according to the projected heat treatment procedure for the sample.

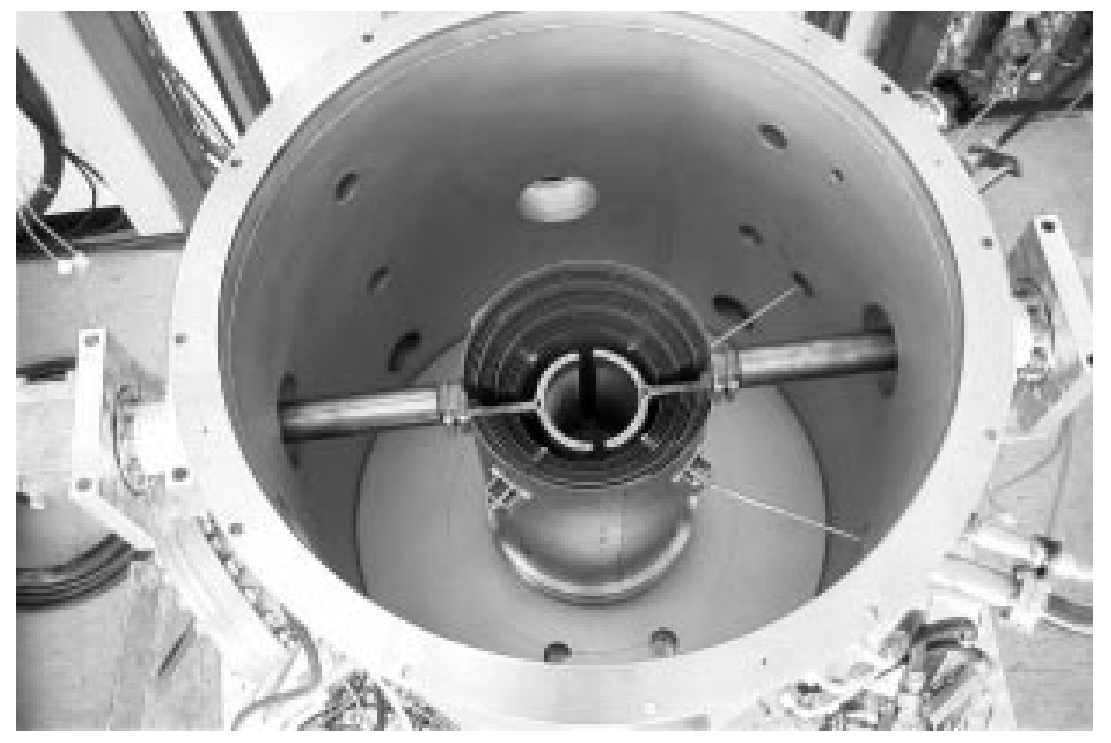

Fig. 3. Photographic view of the furnace, featuring the hot zone with the radiation shield inside the vacuum chamber

\section{Conclusions}

The investigations of the production of neutron rich isotopes from an ion source in-pile have shown the feasibility and the tremendous benefit for RNB experiments. The highest yields with respect to other production methods of neutron rich fission products can be provided for experiments like the production of very neutron rich heavy elements and for investigation of the astrophysical r-process. The efficiency and reliability of the fission fragment production 
depends on the target-ion source combination. Thus a test program has been initiated at Munich in the framework of the MAFF project in order to study the properties of targets and target-ion sources which are most suitable for operation in a high neutron flux.

\section{References}

[1] U. Köster et al. in: Proc. of the international workshop on Research with Fission Fragments, Benediktbeuern, Germany, ed. by T. von Egidy, D. Habs et al. ed., World Scientific, Singapore, 1996, p.29

[2] B. Fogelberg et al., Nucl. Instrum. and Meth. B70 (1992) 137.

[3] D.Habs et al., Munich accelerator for fission fragments, physics case and technical description, Munich, October 1998, (http://www.ha.physik.unimuenchen.de)

[4] R. Smolanczuk, Formation of superheavy elements in cold fusion reactions, Phys. Rev. C 63 (2001) 44607.

[5] H. Ravn et al., Nucl. Instrum. and Meth. B88 (1994) 441.

[6] J.R.J. Bennett et al., Nucl. Instrum. and Meth. B126 (1997) 117.

[7] A.C. Wahl et al., ADNDT 39 (1988) 1.

[8] T. von Egidy et al., Acta physica slovaca 49 (1999) 107.

[9] U. Köster, AIP conference proceedings 475, 1998, p.269

[10] S. Hoffmann et al., Z. Phys. A354 (1996) 229.

[11] Fanny Farget, private communication

[12] R. Silberberg and C. Tsao, Phys. Rep. 191 (1990) 351.

[13] B. Fogelberg, H. Mach, L. Jacobsson, in: Proc. of the international workshop on Research with Fission Fragments, Benediktbeuern, Germany, ed. by T. von Egidy, D. Habs et al. ed., World Scientific, Singapore, 1996, p.69

[14] U. Köster, O. Kester, D. Habs, Rev. Sci. Instrum. 69 (1998) 1316.

[15] U. Köster et al., these proceedings

[16] R. Kirchner, E. Roeckl Nucl. Instrum. and Meth. 133 (1976) 187.

[17] H.J. Maier et al., Nucl. Instrum. and Meth. A438 (1999) 185.

[18] B.D. Bryscin, Advanced Materials and Processes 9 (1992) 22

[19] B.J. Makenas, J.W. Hales and A.L.Ward, AIP Conf. Proc. 212, $8^{\text {th }}$ Space Nuclear Power Systems Conference, Albuquerque 1991, p. 886

[20] H.L. Ravn et al., Nucl. Instrum. and Meth. B26 (1987) 183. 